\title{
Elastic and dynamic properties of membrane phase-field models
}

\author{
Guillermo R. Lázaro ${ }^{1, a}$, Ignacio Pagonabarraga ${ }^{2,3,4}$, and Aurora Hernández-Machado 2,5 \\ 1 Martin Fisher School of Physics, Brandeis University, Waltham, MA, 02454, USA \\ 2 Departament de Fisica de la Matèria Condensada, Universitat de Barcelona, Av. Diagonal 645, E08028 Barcelona, Spain \\ 3 Universitat de Barcelona Institute of Complex Systems (UBICS), Universitat de Barcelona, Barcelona, Spain \\ 4 CECAM, Centre Européen de Calcul Atomique et Moléculaire, École Polytechnique Fédérale de Lasuanne, Batochime, Avenue \\ Forel 2, 1015 Lausanne, Switzerland \\ ${ }^{5}$ Institute of Nanoscience and Nanotechnology (IN2UB), University of Barcelona, Barcelona, Spain
}

Received 18 April 2017 and Received in final form 25 July 2017

Published online: 19 September 2017

(c) The Author(s) 2017. This article is published with open access at Springerlink.com

\begin{abstract}
Phase-field models have been extensively used to study interfacial phenomena, from solidification to vesicle dynamics. In this article, we analyze a phase-field model that captures the relevant physical features that characterize biological membranes. We show that the Helfrich theory of elasticity of membranes can be applied to phase-field models, allowing to derive the expressions of the stress tensor, lateral stress profile and elastic moduli. We discuss the relevance and interpretations of these magnitudes from a phase-field perspective. Taking the sharp-interface limit we show that the membrane macroscopic equilibrium equation can be derived from the equilibrium condition of the phase-field interface. We also study two dynamic models that describe the behaviour of a membrane. From the study of the relaxational behaviour of the membrane we characterize the relevant dynamics of each model, and discuss their applications.
\end{abstract}

\section{Introduction}

Biological membranes are complex objects composed by thousands of molecules, assembled in a delicate balance. The elastic properties of the membrane result from the interplay between all these components, and an astounding number of both passive and active processes are involved in the membrane running [1]. As a consequence of the intrisinc complexity of the membrane microstructure, the mechanical response of cell membranes presents a complex phenomenology which, in spite of the extraordinary insight gained since the beginnings of the field, is still under continuous development. The substantial increase in the quantitative data obtained in recent years invite to a deeper understanding of the membrane functioning by means of physical models, and theoretical studies have provided important information and interpretations of different aspects of the membrane [2,3]. Among others, some relevant examples are the elastic response of the erythrocyte membrane under sedimentation in channel flow [4], the deformability of erythrocytes when manipulated by optical traps [5], or the effect of active processes in the membrane fluctuations [6]. Nevertheless, the study of membrane behaviour is complex, since it usually involves out of equilibrium processes and generic geometries. Thus,

\footnotetext{
a e-mail: grolazaro@gmail.com
}

it requires thermodynamically consistent models characterized by a high flexibility, in order to deal with complicate morphological problems.

From a theoretical perspective, membranes can be treated as interfaces with specific elastic properties, namely a vanishing surface tension and resistance to bend. The dynamics of interfaces is a well-known problem in physics, which has raised in different fields [7]. The socalled phase-field methods have been succesfully applied to study many of these problems, including directed solidification [8], fracture dynamics [9], or roughening [10]. The method consists in introducing an order parameter with two equilibrium phases representing each physical domain, with a smooth interface connecting both regions [11]. The main advantage of the method is that it is not necessary to trace explicitly the interface position. One simply introduces a dynamic equation for the order parameter, and the morphological evolution of the interface is inferred from the order parameter field. The dynamics of the interface will be therefore governed by this dynamic equation, so that the choice of the equation is essential to capture the correct dynamic behaviour of the particular system.

The method was extended to describe amphiphilic systems $[12,13]$, in which the presence of amphiphile molecules lower the surface tension and leads to a more complex interfacial behaviour. Gompper and Zschocke [12] showed that the elastic moduli of the inter- 
face can be estimated from the order parameter profile by comparing the total free energy for simple geometries such as spheres and cylinders. Blokhuis and Bedeaux [14] and Blokhuis [15] demonstrated that the interface elastic response depends on the interface curvature, showing that the properties of curved interfaces might differ significantly from the relaxed state. Taking advantage of the extensive knowledge gathered in phase-field models, it seems natural to extend this method to the modeling of biological membranes [16]. The requirement of a zero surface tension is nonetheless highly nontrivial [17], and different models have been proposed [18-20]. These models have been studied in detail [21], and especially its convergence in a mathematical sense $[22,23]$. However, to date a proper elastic description of phase-field models from the perspective of the membrane framework is lacking. Provided that classic interfaces are much simpler than membranes, the understanding of the elastic properties of membrane phase-field models is essential for a precise control and interpretation of the subsequent studies.

In this paper, we study the membrane phase-field model of ref. [20]. We have two main goals: on the one hand, to show that the mechanic formalism of the classic theory of membranes $[24,25]$ can be applied to phase-field models, allowing to sequentially recover the expressions of the stress tensor, lateral stress profile, and elastic moduli, in terms of the order parameter profile. We compare the results with those found by Gompper and Zschocke [12], following an energetic argument, which we also show here for completeness. By means of a sharp interface limit we then obtain the equilibrium equation of the membrane in the macroscopic limit. On the other hand, we aim to characterize and discuss two different dynamic models and show how the method can be applied to study relaxational processes of the membrane. The paper is structured as follows. In sect. 2 we first briefly outline the Helfrich theory of membranes, and present the specific membrane phasefield model and dynamic equations. In sect. 3 we analyze the relevant elastic properties of the model, obtaining the expressions of the stress tensor, lateral stress profile and elastic moduli. Besides, in sect. 4 we focus on the dynamic characterization of the membrane. We perform a sharp interface limit in order to obtain the macroscopic equations of the membrane. Finally, in sect. 5 we discuss the relevance of the two dynamic models studying the relaxation of a flat membrane by means of a linear stability analysis.

\section{Dynamics of membrane phase-field models}

\subsection{Helfrich theory of membranes}

The large separation in the length scale of the membrane thickness (roughly $4 \mathrm{~nm}$ ) and typical size of the cell $(\approx \mu \mathrm{m})$ allows to consider the membrane as a twodimensional sheet. Based on this assumption, the Helfrich theory establishes that the elasticity of lipid membranes is governed by the resistance to bend, given that bilayers present vanishing surface tension [26] and their fluidic nature in the membrane plane implies that the shear modulus is strictly zero. Thereby, Helfrich [27] proposed the bending free energy,

$\mathcal{F}=\frac{\kappa}{2} \int\left(C-C_{0}\right)^{2} \mathrm{~d} A+\kappa_{G} \int G \mathrm{~d} A+\int \gamma \mathrm{d} A+\int \Delta p \mathrm{~d} V$,

where $C$ and $G$ are the total and Gaussian curvatures of the membrane surface, $\kappa$ and $\kappa_{G}$ are, respectively, the associated bending and Gaussian moduli, and $C_{0}$ is the so-called spontaneous curvature, which captures the presence of any asymmetry in the membrane microstructure. $\gamma$ and $\Delta p$ are two Lagrange multipliers introduced to fix constant membrane area and enclosed volume, respectively. Lipid membranes are highly incompressible [2], due to the strong hydrophobic repulsion of the lipid tails when exposed to water molecules, so that their area remains effectively constant. The constant volume constraint is imposed because certain systems, such as most cells, have specific regulatory systems which maintain the volume (and hence the cell shape) constant. A remarkable aspect of the bending energy (1) concerns the Gaussian term, which remains constant if the membrane deformation does not imply topological changes, as stated by the Gauss-Bonnet theorem.

The Helfrich theory disregards certain aspects of cell membranes, and complementary models have been proposed to improve our physical description of membranes. Most important examples correspond to the bilayer architecture, as captured in the bilayer-couple and areadifference models [28], and the presence of an underlaying spectrin cytoskeleton in the case of red blood cells $[29,30]$. Nevertheless, the Helfrich theory has been the basis to explain an important number of membrane phenomena $[5,31,32]$, and it remains largely valid in general problems in which the cytoskeleton or the balance between leaflets do not play a key role.

\subsection{Phase-field models}

From the phase-field modeling perspective, membranes can be understood as interfaces with two main characteristics: they are tensionless interfaces, and they present resistance to bending. The main challenge in the phasefield modeling of membranes relies on the supression of the surface tension [17]. The bending condition requires the addition of second derivatives of the order parameter in the free energy. Thus, we will consider a general Ginzburg-Landau free energy of the form $[26,33]$

$$
\begin{aligned}
\mathcal{F}[\phi] & =\int \mathcal{L}\left(\phi, \nabla \phi, \nabla^{2} \phi\right) \mathrm{d} V \\
& =\int\left\{f(\phi)+g(\phi)(\nabla \phi)^{2}+c\left(\nabla^{2} \phi\right)^{2}+\mu_{\Delta} \phi\right\} \mathrm{d} V
\end{aligned}
$$

where we have introduced $\mu_{\Delta} \phi$ that accounts for the difference in free energy when the interfacial shape is modified with respect to a reference one. The amplitude $\mu_{\Delta}$ is fixed to enforce that the equilibrium between the two phases in contact through the interface is not affected when 
considering a change in the curvature of the interface. This additional term will only be necessary to derive the elastic coefficients associated to the free energy functional for well-established thermodynamic conditions. For a planar interface $\mu_{\Delta}=0$.

The equilibrium order-parameter profile that minimizes the free energy equation (2) satisfies the EulerLagrange equation

$$
\delta \mathcal{F}=f^{\prime}-2 g \nabla^{2} \phi-g^{\prime}(\nabla \phi)^{2}+2 c \nabla^{2} \nabla^{2} \phi+\mu_{\Delta}=0,
$$

where $\chi^{\prime}(u) \equiv \mathrm{d} \chi / \mathrm{d} u$ denotes the derivative of a generic function $\chi$ with respect to its argument.

\section{A phase-field membrane model}

We make use of a phase-field model that meets the characteristics of membranes $[18,21,22]$, which has been successfully applied to study different problems regarding vesicle and cell elasticity and morphological response [32,34-36]. This model considers the presence of two phases corresponding to the inner and outer regions of the membrane, and the interface separating both domains is specified as a tensionless interface with a bending contribution. Thus, it does not account explicitly for the presence of the lipid-rich phase as a third equilibrium phase, as it is usual in amphiphilic systems [26], but treats the membrane as a two-dimensional sheet with the prescribed elastic behavior. The model corresponds to a free energy of the form (2) with coefficients $f_{b}(\phi)=\phi^{2}-2 \phi^{4}+\phi^{6}$, $g_{b}(\phi)=2 \epsilon^{2}\left(3 \phi^{2}-1\right)$, and $c_{b}=\epsilon^{4}$, where the subscript " $b$ " indicates that it corresponds to a tensionless, purely bending interface. This choice ensures that in equilibrium the surface tension vanishes, as we will show later. The subscript $b$ indicates that this model corresponds to a purely bending interface [20]. Although in principle there are other coefficient sets that produce a vanishing surface tension, the main advantage of this particular choice is that it presents an analytical equilibrium solution of the order parameter profile.

For convenience, we rewrite the free energy as

$$
\mathcal{F}_{b}[\phi]=\int\left\{\frac{\kappa^{*}}{2}(\psi[\phi])^{2}+\mu_{\Delta} \phi\right\} \mathrm{d} V,
$$

where we have introduced the functional $\psi[\phi]=-\phi+\phi^{3}-$ $\epsilon^{2} \nabla^{2} \phi$ and additionally the parameter $\kappa^{*}$ is incorporated to control the bending rigidity of the interface. The equilibrium phases of this model are $\phi_{e q}=0, \pm 1$, though the phase $\phi_{e q}=0$ is not macroscopically stable and hence it is not considered in the subsequent analysis.

In addition to the bending elasticity, the inextensiblity of the membrane can arise as an important contribution to the mechanic response of the membrane. To introduce the Lagrange multiplier $\gamma$ one needs to compute the area of the membrane from the order parameter field. This is achieved exploiting the fact that in the sharp limit $\epsilon \rightarrow 0$, the gradient of the order parameter behaves as a $\delta$-function,

$$
\lim _{\epsilon \rightarrow 0}\left\{\frac{A_{n}(\epsilon)}{2}\left|\nabla_{n} \phi\right|^{2}\right\}=\delta(x),
$$

where $\nabla_{n}$ is the gradient in the normal direction to the interface and $A_{n}(\epsilon)$ is a normalization constant which depends on the specific order parameter profile. This expression allows to transform volume integrals into surface integrals, and hence providing a direct pathway to compute the area of the isosurface $\phi=0$. The complete membrane energy is given by

$$
\mathcal{F}_{\text {mem }}[\phi]=\mathcal{F}_{b}[\phi]+A_{n}(\epsilon) \int \gamma_{A} \epsilon^{2}(\nabla \phi)^{2} \mathrm{~d} V,
$$

where the subscript "mem" indicates that this free energy accounts both for bending and membrane incompressibility. The results presented in the next sections apply to any free-energy of the form eq. (2), although they will allow us to describe and characterize the elastic properties of this phase-field membrane model.

\subsection{Dynamic models}

The Helfrich theory describes the mechanics of lipid membranes, and it can be used to predict equilibrium shapes of cells and vesicles or equilibrium properties of the membrane. However, to study more general out-of-equilibrium problems it must be incorporated in a dynamic framework. The phase-field method inherently invites to introduce a dynamic formalism. The characteristics of the interface dynamics are dictated by the particular choice of the dynamic equation. In this article, we first consider a diffusive dynamics given by the Cahn-Hilliard equation [37],

$$
\frac{\partial \phi}{\partial t}=\nabla \cdot\left(M \nabla \frac{\delta \mathcal{F}}{\delta \phi}\right)
$$

which captures the dynamic behaviour of a membrane immersed in a diffusive medium. Equation (7) is conserved (i.e. the total amount of each equilibrium phase remains constant during the evolution), and therefore the volume multiplier of the membrane energy (1) can be removed. We denote this dynamic model as PF (phase-field).

The behavior of a membrane immersed in a liquid requires to describe the hydrodynamics of the surrounding medium, and thus the Navier-Stokes equation is incorporated,

$$
\begin{aligned}
\frac{\partial \phi}{\partial t}+\mathbf{v} \cdot \boldsymbol{\nabla} \phi & =M \nabla^{2} \frac{\delta \mathcal{F}}{\delta \phi} \\
\rho\left[\frac{\partial \mathbf{v}}{\partial t}+(\mathbf{v} \cdot \nabla \mathbf{v})\right] & =-\nabla P+\mathbf{f}^{\phi}+\eta \nabla^{2} \mathbf{v}
\end{aligned}
$$

Both equations are coupled via the terms $\mathbf{f}^{\phi}$, which represents the force density of the interface disrupting the surrounding fluid, and the advective term $\mathbf{v} \cdot \nabla \phi$, which describes how the fluid accelerates the membrane. The complete Navier-Stokes phase-field model is termed as NSPF. The membrane accelerates locally the fluid through the force density $\mathbf{f}^{\phi}$. We will show in the next section that the local force can be expressed in terms of the chemical potential as

$$
\mathbf{f}^{\phi}=-\phi \nabla \mu .
$$


In order to obtain a close form for the dynamics of the system, we need to identify the stress tensor and chemical potential associated to the phase-field.

\subsection{Chemical potential}

In the phase-field framework the chemical potential is obtained from the variations of the free energy when a small variation in the order parameter is introduced,

$$
\mu[\phi]=\frac{\delta \mathcal{F}}{\delta \phi}=\frac{\partial \mathcal{L}}{\partial \phi}-\nabla_{\beta} \frac{\partial \mathcal{L}}{\partial\left(\nabla_{\beta} \phi\right)}+\nabla^{2} \frac{\partial \mathcal{L}}{\partial\left(\nabla^{2} \phi\right)}+\mu_{\Delta} .
$$

The condition $\mu[\phi]=0$, equivalent to the EulerLagrange eq. (3), determines the equilibrium profile of the order parameter. For the particular case of the membrane model, and considering a flat interface in which the Lagrange multiplier is set to zero, the chemical potential reads

$$
\mu_{m e m}=\kappa^{*}\left[\left(3 \phi^{2}-1\right) \psi[\phi]-\epsilon^{2} \nabla^{2} \psi[\phi]\right] .
$$

From eq. (11) it is obvious that $\psi=0$ represents a solution of the equilibrium condition $\mu_{m e m}=$ const. Considering the boundary conditions $\phi( \pm \infty)= \pm 1$ in the bulk, this expression can be integrated leading to the equilibrium profile $\phi_{0}(z)=\tanh (z / \sqrt{2} \epsilon)$. Although the solution of (11) is not unique, this particular one represents the minimum energy solution, as can be directly inferred from (4). The Lagrange multiplier does not affect the equilibrium profile of the interface, and the previous expression of $\phi_{0}$ represents the equilibrium condition of both free energies (4) and (6). Once the equilibrium profile has been identified, we can compute $A_{n}(\epsilon)=3 /\left(2 \sqrt{2} \epsilon^{2}\right)$ and then the membrane model (6) is completely specified.

\subsection{Stress tensor}

In order to derive the stress tensor, $\sigma_{\alpha \beta}$, from the free energy, eq. (2), we follow a virtual work procedure. Considering a general displacement of the interface given by $\delta x_{\alpha}$, the external work associated reads [38]

$$
\delta \mathcal{F}=-\int \nabla_{\beta} \sigma_{\alpha \beta} \delta x_{\alpha} \mathrm{d} V=\int \sigma_{\alpha \beta} \nabla_{\beta} \delta x_{\alpha} \mathrm{d} V .
$$

The variations of the order parameter associated with this deformation are obtained assuming that the changes induced in the order parameter solely correspond to the interface shift, i.e. diffusion is subdominant [39],

$$
\partial_{t} \phi+\nabla \cdot(\phi \mathbf{v})=0
$$

and considering $v_{\alpha} \approx \delta x_{\alpha} / \delta t$, we derive the variation of the order parameter and, after differentiation, its deriva- tives

$$
\begin{aligned}
\delta \phi= & -\phi \nabla_{\alpha} \delta x_{\alpha}-\nabla_{\alpha} \phi \delta x_{\alpha} . \\
\delta \nabla_{\beta} \phi= & -\nabla_{\beta} \phi \nabla_{\alpha} \delta x_{\alpha}-\phi \nabla_{\beta} \nabla_{\alpha} \delta x_{\alpha} \\
& -\nabla_{\beta} \nabla_{\alpha} \phi \delta x_{\alpha}-\nabla_{\alpha} \phi \nabla_{\beta} \delta x_{\alpha} . \\
\delta \nabla^{2} \phi= & -\nabla^{2} \phi \nabla_{\alpha} \delta x_{\alpha}-2 \nabla_{\beta} \phi \nabla_{\beta} \nabla_{\alpha} \delta x_{\alpha}-\phi \nabla_{\alpha} \nabla^{2} \delta x_{\alpha} \\
& -\nabla_{\alpha} \nabla^{2} \phi \delta x_{\alpha}-2 \nabla_{\beta} \nabla_{\alpha} \phi \nabla_{\beta} \delta x_{\alpha}-\nabla_{\alpha} \phi \nabla^{2} \delta x_{\alpha} .
\end{aligned}
$$

Thereby, the work required to deform the interface reads

$$
\begin{aligned}
\delta \mathcal{F} & =\int \delta \mathcal{L} \mathrm{d} V \\
& =\int\left(\frac{\partial \mathcal{L}}{\partial \phi} \delta \phi+\frac{\partial \mathcal{L}}{\partial \nabla_{\beta} \phi} \delta \nabla_{\beta} \phi+\frac{\partial \mathcal{L}}{\partial \nabla^{2} \phi} \delta \nabla^{2} \phi\right) \mathrm{d} V
\end{aligned}
$$

Introducing the variations of the order parameter and its gradients, (14), in the expression (15), and after several straightforward integrations of those terms containing second and third gradients of $\delta x_{\alpha}$, one obtains the stress tensor,

$$
\begin{aligned}
\sigma_{\alpha \beta}= & \left(\mathcal{L}-\phi \frac{\delta \mathcal{L}}{\delta \phi}\right) \delta_{\alpha \beta}-\frac{\partial \mathcal{L}}{\partial\left(\nabla_{\beta} \phi\right)} \nabla_{\alpha} \phi \\
& +\left(\nabla_{\alpha} \phi\right) \nabla_{\beta} \frac{\partial \mathcal{L}}{\partial\left(\nabla^{2} \phi\right)}-\frac{\partial \mathcal{L}}{\partial\left(\nabla^{2} \phi\right)} \nabla_{\alpha} \nabla_{\beta} \phi .
\end{aligned}
$$

The force exerted by an object is defined as the divergence of the stress tensor. By directly operating in eq. (16), it can be shown that for an interface with free energy (2), in general the force density relates with the chemical potential by

$$
f_{\alpha}^{\phi}=\nabla_{\beta} \sigma_{\alpha \beta}=-\phi \nabla_{\alpha} \mu .
$$

For the particular case where the free energy corresponds to a membrane, eq. (6), the previous general expression for the local force due to the phase-field reduces to eq. (9).

\section{Elastic properties of membrane phase-field models}

\subsection{Lateral stress profile. Mechanical derivation of the membrane elastic moduli}

The lateral stress profile of an interface is obtained from the stress tensor [40],

$$
s(z)=\sigma_{t}-\sigma_{n},
$$

where $\sigma_{t}$ and $\sigma_{n}$ denote the tangential and normal components of the stress tensor, respectively. Assuming a flat interface along the $x y$ plane, these components reduce to $\sigma_{n}=\sigma_{z z}$ and $\sigma_{t}=(1 / 2)\left(\sigma_{x x}+\sigma_{y y}\right)$, and the stress profile is easily computed from (16),

$$
s_{\phi}(z)=2 g\left(\phi_{0}\right)\left(\phi_{0}^{\prime}\right)^{2}+2 c\left(\phi_{0}^{\prime \prime}\right)^{2}-2 c \phi_{0}^{\prime \prime \prime} \phi_{0}^{\prime} .
$$






Fig. 1. (A) Scheme of a lipid bilayer. The multiple interactions between the chemical groups of the lipids lead to a complex stress profile in real cell membranes. However, bilayers are invariably characterized by a balance between internal stresses, giving rise to a vanishing surface tension. (B) Lateral stress profile of the membrane phase-field model, $s_{\phi}^{b}(z)$ (blue line), and for a classic tension interface, $s_{\phi}^{t}(z)$ (red line). The phase-field model for membranes does not account for a detailed profile of the actual stresses present in the membrane, but concentrates the interactions in two effective contributions, a central term of repulsion and two symmetric attractions, and their balance produces the tensionless nature of the membrane. The lateral stress profile of a tension interface, on the contrary, involves a single term of attraction, so that the interface tries to minimize its surface.

The internal stresses dictate the macroscopic elastic properties of the interface [25]. In fig. 1 we show the lateral stress profile for a tension interface (e.g., a classic wateroil interface) and a membrane (given by the free energy model (4)). The tension interface has a unique positive contribution, implying that the pressure is negative and thus the interface tends to compress, minimizing its surface area. The profile of the membrane is more complex, as it presents a central negative term (which following an analogous argument can be interpreted as a repulsion) and two symmetric terms of attraction. One of the main important aspects of this profile is that the different stresses are balanced, so that its integral along $\hat{z}$ is zero.

Lateral stress profiles have been extensively studied in membranes, both theoretically [41] and numerically [42], providing a deeper understanding of the interactions between lipids or the influence of the presence of proteins to the lateral balance of the membrane [43]. Studies at a molecular level have shown that the lateral stresses, even in simple homogeneous membranes, present a complex profile [44], resulting from the numerous chemical interactions, such as repulsion between polar groups, hydrophobic attraction, repulsive effects between tails, etc. Therefore, the stress profile of the phase-field model of fig. 1 might be understood as an effective profile which recovers the fundamental macroscopic properties. Still, we offer here an alternative interpretation of this profile. The central repulsive term can be understood as the entropic repulsion between lipid tails at the central core of the membrane, which try to expand the membrane. The two lateral attractions must be associated to the head-tail interactions, which compress the membrane. Accordingly, the phase-field approach implicitly entails a simple representation of the membrane internal molecular interactions which follows the spirit of the initial simple descriptions by Petrov and Bivas [45]. In principle, the phase-field framework might allow to construct more detailed models, in which the presence of the lipid phase is considered and hence the correspondent lateral stresses would reproduce a more realistic profile.

According to Helfrich [24], the elastic moduli that characterize the membrane elastic energy, eq. (1), can be obtained as moments of the lateral stress profile. Following his approach, the surface tension, spontaneous curvature and Gaussian curvature modulus read

$$
\begin{aligned}
\bar{\gamma}^{m e c} & =\int s_{\phi}(z) \mathrm{d} z, \\
-\kappa C_{0}^{m e c} & =\int z s_{\phi}(z) \mathrm{d} z, \\
\kappa_{G}^{m e c} & =\int z^{2} s_{\phi}(z) \mathrm{d} z,
\end{aligned}
$$

where we have introduced $\bar{\gamma}^{m e c}=\gamma+(\kappa / 2)\left(C_{0}^{m e c}\right)^{2}$. As opposed to eqs. (20)-(21), previous studies have shown that eq. (22) is not generally valid [46].

\subsection{Energetic derivation of the elastic moduli}

Alternatively, it is possible to derive the expressions for the elastic moduli comparing the membrane free energy in the phase-field representation with the corresponding expressions of the Helfrich energy of spheres and cylinders $[12-14,47]$. We outline here the main steps of the derivation, but the complete process is shown in appendix A. If we consider a membrane with spherical or cylindrical shape, the Helfrich energy reads

$$
\begin{aligned}
& \frac{F_{c}}{A}=\bar{\gamma}-\frac{\kappa C_{0}}{R}+\frac{\kappa}{2 R^{2}} \\
& \frac{F_{s}}{A}=\bar{\gamma}-\frac{2 \kappa C_{0}}{R}+\frac{2 \kappa+\kappa_{G}}{R^{2}} .
\end{aligned}
$$

If we consider a membrane with cylindrical and spherical geometry in which the radius is very large, the radial coordinate close to the interface matches the normal component of a flat membrane, $z=r-R$. Exploiting this symmetry, the membrane free energy, eq. (2) can be expressed in terms of the energy per unit area as an integral along the radial coordinate,

$$
\frac{\mathcal{F}_{c / s}}{A}=\int \mathrm{d} z\left(1+\frac{z}{R}\right)^{d} \mathcal{L}_{c / s}\left(\phi, \nabla \phi, \nabla^{2} \phi\right),
$$

where $c / s$ refers to the membrane geometry (cylinder or sphere, respectively) and $d=1(2)$ for the cylinder (sphere). $\mathcal{L}$ has units of pressure and is related to the excess of pressure across the interface, as we will describe.

In the limit in which the radius of curvature is large enough, we can expand the different contributions to eq. (24) in powers of $1 / R$. For example, the order parameter can be expressed as

$$
\phi_{c / s}(r-R)=\phi_{0}(z)+\frac{\phi_{1, c / s}(z)}{R}+\frac{\phi_{2, c / s}(z)}{R^{2}}+\mathcal{O}\left(R^{-3}\right)
$$


where the coefficients of the expansion are functions that depend on the membrane geometry, except the zeroth order $\phi_{0}$, which corresponds to the reference, planar profile. Analogously, for the free energy we can write

$$
\begin{aligned}
\frac{\mathcal{F}_{c / s}}{A}= & \int \mathrm{d} z\left(1+\frac{z}{R}\right)^{d} \\
& \times\left[\mathcal{L}_{0}+\frac{\mathcal{L}_{1, c / s}}{R}+\frac{\mathcal{L}_{2, c / s}}{R^{2}}+\mathcal{O}\left(R^{-3}\right)\right],
\end{aligned}
$$

where the functions $\mathcal{L}_{0}=\mathcal{L}\left(\phi_{0}\right), \mathcal{L}_{1, c / s}=\partial \mathcal{L}_{c / s} / \partial(1 / R)$, and $\mathcal{L}_{2, c / s}=\partial^{2} \mathcal{L}_{c / s} / \partial(1 / R)^{2}$ correspond to the amplitudes of the free energy density in subsequent powers of the radius of curvature. Comparing the expansion in the free energy, eq. (26), with the Helfrich free energies, eq. (23), when the interface forms a sphere and a cylinder, we can express the mechanical properties of the interface in terms of the expansion of the free energy density in the phase-field approach. Specifically, for a cylindrical deformation we obtain

$$
\begin{aligned}
\bar{\gamma} & =\int \mathcal{L}_{0} \mathrm{~d} z \\
-\kappa C_{0} & =\int\left[z \mathcal{L}_{0}+\mathcal{L}_{1, c}\right] \mathrm{d} z \\
\kappa & =2 \int\left[z \mathcal{L}_{1, c}+\mathcal{L}_{2, c}\right] \mathrm{d} z,
\end{aligned}
$$

while for a spherical deformation we arrive at

$$
\begin{aligned}
\bar{\gamma} & =\int \mathcal{L}_{0} \mathrm{~d} z \\
-2 \kappa C_{0} & =\int\left[2 z \mathcal{L}_{0}+\mathcal{L}_{1, s}\right] \mathrm{d} z \\
2 \kappa+\kappa_{G} & =\int\left[z^{2} \mathcal{L}_{0}+2 z \mathcal{L}_{1, s}+\mathcal{L}_{2, s}\right] \mathrm{d} z .
\end{aligned}
$$

The subscripts $c$ and $s$ indicate that the corresponding amplitude is obtained by an expansion of $\mathcal{F}$, in the inverse of the curvature radius for a spherical and cylindrical shape, respectively. Since the lowest order in the expansion corresponds to the reference, planar interface, $\mathcal{L}_{0}$ is independent of the deformed interface, and correspondingly, the expression for the surface tension obtained is the same for a spherical and cylindrical deformation, and reads

$$
\mathcal{L}_{0}=f_{0}+g_{0}\left(\phi_{0}^{\prime}\right)^{2}+c\left(\phi_{0}^{\prime \prime}\right)^{2} .
$$

Multiplying the Euler-Lagrange expression, eq. (3), by the gradient of the order parameter for a planar interface we get

$$
f_{0}=g_{0}\left(\phi_{0}^{\prime}\right)^{2}+c\left(\phi_{0}^{\prime \prime}\right)^{2}-2 c \phi_{0}^{\prime} \phi_{0}^{\prime \prime \prime},
$$

except for a constant that we take 0 to avoid divergences in the bulk phases. Inserting this constraint in eq. (29) shows that the integral across the interface of the profiles $\mathcal{L}_{0}$ and $s_{\phi}$ are equivalent, indicating that the surface free energy for a planar interface coincides with the excess pressure across the interface. Hence the surface tension obtained from eqs. (28)-(27) and eq. (20) coincide. The same holds for the product $\kappa C_{0}$, as shown in appendix $\mathrm{A}$.

A cylindrical deformation provides a relation between the bending modulus and $\mathcal{L}_{1, c}$ and $\mathcal{L}_{2, c}$. In order to analyze the dependence of $\kappa$ on the properties of the free energy density one needs to perform the expansion of the free energy density to second order in the cylinder curvature, and select a dividing surface. The derivations corresponding to the equimolar surface, for which $\int \mathrm{d} z\left[\phi(z)-\phi_{\text {bulk }}(z)\right]=0$, is carried out in appendix $\mathrm{A}$, leading to

$$
\kappa=\int 2 c\left(\phi_{0}^{\prime}\right)^{2} \mathrm{~d} z-\int\left[g_{0} \phi_{0}^{\prime}-2 c \phi_{0}^{\prime \prime \prime}\right] \phi_{1, c} \mathrm{~d} z .
$$

The first term of this expression only depends on the planar reference profile, and actually is proportional only to the amplitude $c$ in the free energy density, in agreement with the result found in [12]. The second term represents the contribution to the rigidity due to the bending of the interface, as determined by the presence of the perturbative term $\phi_{1, c}$. Blokhuis [15] discussed to what extent the expression given by [12] (which we denote as $\left.\kappa_{0}=\int 2 c\left(\phi_{0}^{\prime}\right)^{2} \mathrm{~d} z\right)$ is valid, or whether the whole expression eq. (31) recovers the bending rigidity. He showed that the approximation $\kappa_{0}$ is in general incorrect, and can lead to large deviations from the real value. However, for some specific free energies, such as that used by [12], the perturbative term associated with $\phi_{1, c}$ is very small, and hence the bending rigidity calculation based only on the planar profile is sufficiently accurate, $\kappa \sim \kappa_{0}$. For the free energy model corresponding to eq. (2) we have verified that $\kappa_{0} / \kappa=0.98$, thus showing that $\kappa_{0}$ constitutes a good estimate of the bending rigidity.

Finally, we can derive an expression for the Gaussian curvature from the spherical deformation of the free energy model. The expression appearing in eq. (28) indicates that in general we cannot recover the mechanical expression proposed by Helfrich, depicted in eq. (22). The mechanical expression can be recovered only if $\int\left(\mathcal{L}_{2, s}+\right.$ $\left.2 z \mathcal{L}_{1, s}\right) \mathrm{d} z=0$. This is generically not the case. For the particular case of a liquid interface, when $c=0$ and $g(\phi)=g_{0}$ this expression cancels and the mechanical and energetic expressions for the Gaussian curvature coincide. The Gaussian curvature can then be understood as a moment of the excess stress along the unperturbed interface. For the general case we get

$$
\kappa_{G}=\int\left(z^{2}\left[2 g(\phi)\left(\phi_{0}^{\prime}\right)^{2}+4 c\left(\phi_{0}^{\prime \prime}\right)^{2}\right]-4 c\left(\phi_{0}^{\prime}\right)^{2}\right) \mathrm{d} z,
$$

as derived in the appendix A. The mechanical derivation gives as obtained from eqs. (22)-(22) gives

$$
\kappa_{G}^{(m e c)}=\int\left(z^{2}\left[2 g(\phi)\left(\phi_{0}^{\prime}\right)^{2}+4 c\left(\phi_{0}^{\prime \prime}\right)^{2}\right]-2 c\left(\phi_{0}^{\prime}\right)^{2}\right) \mathrm{d} z .
$$

For a lipidic symmetric membrane, consistent with the expected result of a purely bending interface, we recover the expected results for the surface tension, spontaneous curvature and bending rigidity, $\gamma=C_{0}=0$, 
$\kappa=\left(2 \sqrt{2} \epsilon^{3} / 3\right) \kappa^{*}$. A vanishing Gaussian curvature, according to the theoretical model [21], is only recovered from the energetic expression, eq. (32). Hence, there is an inconsistency in the Gaussian rigidity between the mechanical expression proposed by Helfrich and the value obtained from energetic arguments based on the equimolar dividing surface Previous studies have already shown that eq. (22) does not hold for a general phase-field model for surfactants [46]. Although the discrepancy in the Gaussian curvature for these models is small, this difference may explain the deviations observed when comparing Gaussian modulus computed numerically from molecular level simulations of lipidic membranes, as discussed by $\mathrm{Hu}$ et al. [44]. These authors compare the value of the Gaussian modulus obtained from the second moment of the lateral stress profile with the value derived when changes in the topology of the membrane are induced, finding that the mechanic approach leads to unphysical results and concluding that the mechanical approach based on the moments of the profile does not correctly capture important correlations within the bilayer. A more detailed analysis of the relationship between the stress tensor obtained from the Ginzburg-Landau free energy and its microscopic derivation is required. In particular, it is unclear which aspects of the details of the location of the curved interface at a microscopic level will be crucial to identify the proper bending rigidities.

\section{Macroscopic equations}

The results presented in the previous section allow to connect the macroscopic mechanical properties of the interface with the gradient profiles of the order parameter. This fact can be exploited to derive the macroscopic equations of the interface from the equations for the order parameter, (10) and (7). This procedure, known as the sharp interface limit, has been extensively applied in phase-field models to obtain the set of equations that describe the dynamics of the interface $[19,48]$, allowing the study of some important interfacial behaviour such as the SaffmanTaylor instability [49]. The goal of this section is to apply the sharp-interface method to a general membrane phasefield model of the form eq. (2) and obtain the macroscopic equations that characterize the equilibrium and dynamics of this model.

The sharp interface limit consists in considering a macroscopic length scale, much larger than $\epsilon$, and derive the equations that depend on the macroscopic variables, such as the total curvature of the interface, in the limit $\epsilon \rightarrow 0$. It is a singular limit, as $\epsilon$ must be small but strictly nonzero. We first focus on the dynamic equation of the order parameter without hydrodynamic coupling, PF. We then discuss the application to the complete NS-PF model.

We only outline the sharp interface limit here, but further details of the method can be found in appendix B. The method is mathematically analogous to the expansion in $1 / R$ carried out in the previous section, but following the standard procedure it is generalized to account for any interface geometry, instead of being limited to cylindrical and spherical surfaces. For that purpose, we deploy curvilinear coordinates. The space is separated into two regions, the (macroscopic) outer region, and the (microscopic) inner region of the interface. Both regions are described by the coordinates $r$ (see footnote ${ }^{1}$ ) and $\omega$, respectively, related by $r=\omega / \epsilon$. Thus, the fast coordinate in the inner region allows to resolve the details of the interface. In the macroscopic scale, the interface is sharp and then $\epsilon \rightarrow 0$. The starting point is a flat interface in equilibrium, subjected to a weak perturbation. The interface is characterized by the general free energy (2). The interface must be symmetric, $c_{0}=0$, so that the flat interface represents an equilibrium configuration; for strictly nonsymmetric interfaces the method should perturb an equilibrium configuration, such as a spherical membrane of radius $1 / c_{0}$, requiring a more complex analytical treatment. Once the fast coordinates are introduced, as explained in appendix B, the dynamic equation reads

$$
\epsilon \partial_{\tau} \phi-\frac{1}{\epsilon} v \partial_{\omega} \phi=M\left(\frac{1}{\epsilon^{2}} \partial_{\omega}^{2}-\frac{C}{\epsilon} \partial_{\omega}+\partial_{s}^{2}+\partial_{u}^{2}\right) \mu,
$$

where $v$ denotes the normal velocity of the interface.

\subsection{Membrane equilibrium equation}

The equilibrium equation of a Helfrich membrane was first derived by Ou-Yang and Hefrich [50] applying a variational argument. Following a different procedure, the sharp interface limit allows to derive this macroscopic equation [22], imposing the condition $\mu=$ const. (Note that this condition directly gives $v=0$ in (34)). The problem reduces then to the calculation of this constant in terms of the macroscopic variables. For the equilibrium flat interface, the chemical potential vanishes $\mu_{0}=0$ but for other geometries its value is a priori unknown. As mentioned, the method assumes a weak perturbation of the reference flat interface. The phase-field profile of the perturbed interface is expanded

$$
\phi=\phi_{0}+\epsilon \phi_{1}+\epsilon^{2} \phi_{2}+\ldots,
$$

where $\phi_{0}$ is the equilibrium profile. Functionals of $\phi$ are expanded as $m\left(\phi_{0}+\epsilon \phi_{1}+\epsilon^{2} \phi_{2}+\ldots\right)=m_{0}\left(\phi_{0}\right)+$ $\epsilon m_{1}\left(\phi_{0}, \phi_{1}\right)+\epsilon^{2} m_{2}\left(\phi_{0}, \phi_{1}, \phi_{2}\right)+\ldots$ Likewise, the differential operators are expanded in terms of $\epsilon$, as described in appendix B. Using these results, the chemical potential for the free energy model introduced in eq. (2) can be explicitly derived from eq. (10) leading to

$$
\begin{aligned}
\mu_{0}= & f_{0}^{\prime}-g_{0}^{\prime}\left(\partial_{\omega} \phi_{0}\right)^{2}-2 g_{0} \partial_{\omega}^{2} \phi_{0}+2 c \partial_{\omega}^{2} \phi_{0} \\
\mu_{1}= & f_{1}^{\prime}-g_{1}^{\prime}\left(\partial_{\omega} \phi_{0}\right)^{2}-2 g_{1} \partial_{\omega}^{2} \phi_{0}+2 C g_{0} \partial_{\omega} \phi_{0}-2 g_{0} \partial_{\omega}^{2} \phi_{1} \\
& +2 c \partial_{\omega}^{4} \phi_{1}-4 c C \partial_{\omega}^{3} \phi_{0} \\
& \ldots
\end{aligned}
$$

${ }^{1} r$ represents the direction normal to the interface in the curvilinear coordinates, as described in detail in appendix B. For the specific case of a flat interface (such as the equilibrium one, $\left.\phi_{0}\right)$, this coordinate agrees with $z$ as used in sect. 3 . The coordinates along the membrane surface are $(s, u)$. 
where $f_{1}$ and $g_{1}$ represent the terms of order $\epsilon_{1}$ of the expanded functions $f$ and $g$, respectively, and we use the notation $f^{\prime}=\partial f / \partial \phi$ and $f_{0}=f\left(\phi_{0}\right)$. The equilibrium value, $\mu_{0}=0$ provides the equilibrium phases $\phi_{e q}$ and defines the equation for $\phi_{0}$. The next contribution $\mu_{1}$ is nonzero, and its value is evaluated by multiplying by $\partial_{\omega} \phi_{0}$ and integrating over the normal direction,

$$
\begin{aligned}
\mu_{1} \int \mathrm{d} \omega \partial_{\omega} \phi_{0}= & \int \mathrm{d} \omega\left[f_{1}^{\prime}-g_{1}^{\prime}\left(\partial_{\omega} \phi_{0}\right)^{2}-2 g_{1} \partial_{\omega}^{2} \phi_{0}\right. \\
& +2 C g_{0} \partial_{\omega} \phi_{0}-2 g_{0} \partial_{\omega}^{2} \phi_{1} \\
& \left.+2 c \partial_{\omega}^{4} \phi_{1}-4 c C \partial_{\omega}^{3} \phi_{0}\right] \partial_{\omega} \phi_{0} .
\end{aligned}
$$

The right-hand side of this expression includes terms depending solely on $\phi_{0}$ whereas others also depend on $\phi_{1}$. We focus first on the latter. By using the relation $m_{1}^{\prime}=m_{0}^{\prime \prime} \phi_{1}$ for any polynomial function $m,(37)$ can be rewritten into

$$
\begin{aligned}
\mu_{1} \int \mathrm{d} \omega \partial_{\omega} \phi_{0}= & \int \mathrm{d} \omega \partial_{\omega} \phi_{0}\left[f_{0}^{\prime \prime}-g_{0}^{\prime \prime}\left(\partial_{\omega} \phi_{0}\right)^{2}\right. \\
& \left.-2 g_{0}^{\prime \prime} \partial_{\omega}^{2} \phi_{0}-2 g_{0}^{\prime} \partial_{\omega}^{2}+2 c \partial_{\omega}^{4}\right] \phi_{1} \\
& +\int \mathrm{d} \omega \partial_{\omega} \phi_{0}\left[2 C g_{0} \partial_{\omega} \phi_{0}-4 c C \partial_{\omega}^{3} \phi_{0}\right] .
\end{aligned}
$$

Let us consider now the equation that determines the equilibrium profile, given by $\mu_{0}=0$. Applying the operator $\partial_{\omega}$, we obtain the equation

$$
\left[f_{0}^{\prime \prime}-g_{0}^{\prime \prime}\left(\partial_{\omega} \phi_{0}\right)-2 g_{0}^{\prime \prime} \partial_{\omega}-2 g_{0}^{\prime} \partial_{\omega}+2 c \partial_{\omega}^{3}\right] \partial_{\omega} \phi_{0}=0 .
$$

Thus, $\partial_{\omega} \phi_{0}$, known as Goldstone mode and related to the translational invariance of the interface, is an eigenvector of the linear operator in brackets in (39) [49]. Integrating by parts (38) and introducing (39), the term associated to $\phi_{1}$ vanishes and then (37) reduces to

$$
\mu_{1} \int \mathrm{d} \omega \partial_{\omega} \phi_{0}=\int \mathrm{d} \omega\left[2 C g_{0}\left(\partial_{\omega} \phi_{0}\right)^{2}-4 c C \partial_{\omega}^{3} \phi_{0} \partial_{\omega} \phi_{0}\right]
$$

Integrating by parts, this equation reads

$$
\mu_{1} \phi(+\infty)-\mu_{1} \phi(-\infty)=C \int \mathrm{d} \omega s_{\phi}(\omega)
$$

where we have introduced the lateral stress profile (19).

To relate the gradients of the chemical potential with the corresponding pressure gradients at the two sides of the membrane, we introduce the pressure tensor $P_{\alpha \beta}=$ $-\sigma_{\alpha \beta}$ and consider eq. (17), arriving at $\partial_{r} p=\phi_{e q} \partial_{r} \mu$ (see footnote $\left.{ }^{2}\right)$. Since $\phi=\phi( \pm \infty)$ is constant far from the interface, in the macroscopic scale we can relate the pressure

2 This relation can be understood from the Gibbs-Duhem equation,

$$
V \mathrm{~d} P=\sum_{i} N_{i} \mathrm{~d} \mu_{i}
$$

where $N_{i}$ is the amount of matter of the species $i$ and taking into account that $\phi \sim N / V$, which leads to $\mathrm{d} P=\phi \mathrm{d} \mu$. and chemical potential as $p_{1}( \pm 0)=\phi_{e q} \mu_{1}( \pm 0)$ [49]. Using this relation and the expression for the surface tension, eq. (20), in eq. (41) we arrive at

$$
\Delta p_{1}=\gamma C
$$

which corresponds to the Young-Laplace equation that provides the pressure difference sustained across the interface.

By means of this procedure, the subsequent terms of the chemical potential can be evaluated, providing highorder contributions to the pressure difference. The explicit calculations are simple but cumbersome, and only the main steps are shown here. By using the equation $\partial_{w} \mu_{1}=0$ as with the Goldstone mode, evaluation of $\mu_{2}$ reduces to

$$
\begin{aligned}
\mu_{2} \int \mathrm{d} \omega \partial_{\omega} \phi_{0}= & \left(C^{2}-2 G\right) \\
& \times \int \mathrm{d} \omega \omega\left[2 g_{0}\left(\partial_{\omega} \phi_{0}\right)^{2}-4 c \partial_{\omega}^{3} \phi_{0} \partial_{\omega} \phi_{0}\right]
\end{aligned}
$$

The term of the right-hand side of this expression vanishes, since $s(\omega)$ is strictly symmetric. The term $\Delta p_{2}$ corresponds to the pressure contribution of the spontaneous curvature and accordingly it vanishes for a symmetric membrane. From the algebraic calculations shown in appendix B, and considering the final expression (B.19), the subsequent term is given by

$$
\begin{aligned}
\mu_{3} \int \mathrm{d} \omega \partial_{\omega} \phi_{0}= & \left(C^{3}-3 G C\right) \int \mathrm{d} \omega \omega^{2}\left[2 g_{0}\left(\partial_{\omega} \phi_{0}\right)^{2}\right. \\
& \left.-4 c \partial_{\omega}^{3} \phi_{0} \partial_{\omega} \phi_{0}\right]-\Delta_{s} C \int \mathrm{d} \omega 2 c\left(\partial_{\omega} \phi_{0}\right)^{2} \\
& -\frac{1}{2} C\left(C^{2}-4 G\right) \int \mathrm{d} \omega 2 c\left(\partial_{\omega} \phi_{0}\right)^{2}
\end{aligned}
$$

The first term in the right-hand side of (45) vanishes due to the symmetry of the equilibrium profile, and identifying the bending rigidity from (31), we obtain

$$
\Delta p_{3}=-\frac{1}{2} \kappa C\left(C^{2}-4 G\right)-\kappa \Delta_{s} C .
$$

Considering all the contributions and disregarding for simplicity the term associated to the Gaussian curvature modulus, the membrane equilibrium equation reads

$\Delta p=\Delta p_{0}+\epsilon \Delta p_{1}+\ldots=\gamma C-\frac{1}{2} \kappa C\left(C^{2}-4 G\right)-\kappa \Delta_{s} C$.

\subsection{Dynamic equations}

In the previous section we have restricted our analysis to equilibrium, disregarding the dynamics described by eq. (7). The sharp interface limit can be extended to obtain the macroscopic equations that describe the dynamic 
behaviour of the membrane. The derivation of the complete dynamic model is necessary to study, among others, the stability and relaxational properties of the interface.

Following the formalism presented in the previous section, the space is separated in two domains, the inner corresponding to the interfacial region and the outer, which corresponds to the bulk. The variables of eq. (7) are expanded in terms of $\epsilon$. In the inner region, the order $\epsilon^{-2}$ is given by $\partial_{\omega}^{2} \mu_{0}=0$, and hence $\mu_{0}=m_{0}+n_{0} \omega$. Since $\mu_{0}$ must be finite in the limit $\omega \rightarrow \pm \infty, n_{0}$ vanishes and we fix $\mu_{0}=0$. The dynamic equation at order $\epsilon^{-1}$ reads

$$
-v_{0} \partial_{\omega} \phi_{0}=M \partial_{\omega}^{2} \mu_{1}
$$

Integrating this equation across the interface profile,

$$
\partial_{\omega} \mu_{1}(+\infty)-\partial_{\omega} \mu_{1}(-\infty)=-v_{0} \Delta \phi_{e q} .
$$

We arrive at $v_{0}=0$ introducing the matching conditions $\partial_{\omega} \mu_{1}( \pm \infty)=\partial_{r} \mu_{0}( \pm 0)=0 . \mu_{1}$ is therefore a constant that can be evaluated as in the previous section. The subsequent order, $\epsilon^{0}$, is given by

$$
-v_{1} \partial_{\omega} \phi_{0}=M\left(\partial_{\omega}^{2} \mu_{2}-C \partial_{\omega} \mu_{1} \partial_{s}^{2} \mu_{0}\right) .
$$

Integration by parts and using the matching conditions lead to

$$
\begin{aligned}
-\Delta \phi_{e q} v_{1} & =M\left(\partial_{\omega} \mu_{2}(+\infty)-\partial_{\omega} \mu_{2}(-\infty)\right) \\
& =M\left(\partial_{r} \mu_{1}(+0)-\partial_{r} \mu_{1}(-0)\right) .
\end{aligned}
$$

We can use again the relation between pressure and chemical potential gradients, eq. (17) to arrive at an explicit expression for the membrane velocity. Taking into account that $\left|\phi_{e q}\right|=1$ and introducing the permeability $B=M /\left(2\left|\phi_{e q}\right|^{2}\right),(51)$ can be rewritten,

$$
v=-B\left(\nabla p^{+}+\nabla p^{-}\right) .
$$

This is the continuity equation that describes the interface velocity as a function of the pressure gradients at the interface.

In the outer region, at first order $\mu_{0}=0$ due to the matching conditions with the inner region. The subsequent order, however, is given by $\nabla^{2} \mu_{1}=0$ which can be rewritten as $\nabla^{2} p=0$. This expression represents the Laplace equation in the bulk.

Taking into account the three equations obtained, the complete macroscopic model reads

$$
\begin{aligned}
\Delta p & =\gamma C-(1 / 2) \kappa C\left(C^{2}-4 G\right)-\kappa \Delta_{s} C, \\
v & =-B\left(\nabla p^{+}+\nabla p^{-}\right), \\
\nabla^{2} p & =0 .
\end{aligned}
$$

Some remarks should be pointed out here. Although we have included the equilibrium equation (47) for completeness, the sharp interface limit specifies that the dynamics is dominated by the first contribution to the pressure difference. Thus, if the interface has a nonzero surface tension, its dynamics will be generally dictated by surface tension. For symmetric tensionless interfaces, in which the first nonvanishing term is $\Delta p_{3}$, bending governs the dynamics of the interface.

\section{Membrane relaxation dynamics}

The macroscopic model (53) describes the dynamics of the membrane for the PF model. In deterministic dynamics, a linear stability analysis can be performed to obtain information about the properties and relaxational behaviour of the membrane, analogous to the study of the fluctuation spectrum in stochastic systems, as stated by the Onsager's theory of linear relaxation processes. We perform a linear stability analysis to study the response of a flat interface in equilibrium when subjected to a sinusoidal perturbation [51]. The relaxational time of the membrane is calculated, and the method is applied to measure the elastic properties of the membrane. The flat interface is weakly perturbed, and the membrane position is then given by $y=h(x, t)=\xi_{0} e^{i q x+\omega t}$, where $h$ is the distance of the membrane with respect to the equilibrium position, $q$ is the wavelength of the perturbation and $\omega$ is the relaxation rate. If $\omega>0$, the perturbation will grow and the membrane is unstable; if $\omega<0$, the membrane is stable and it recovers the initial configuration in a typical relaxation time $1 / \omega$. For the pressure field we assume the ansatz $p=p_{0}+A e^{i q x+k y+\omega t}$, where $1 / k$ is the distance from the interface in which the pressure converges to the bulk value. For sufficiently small amplitudes, the curvature of the interface can expressed as $C \approx-\partial_{x}^{2} \xi=q^{2} \xi$. Finally, the velocity of the interface is given by $v=\partial_{t} h$. Introducing all these considerations into eq. (53), we find

$$
\begin{aligned}
A & =\left(\gamma q^{2}+\kappa q^{4}\right) \xi, \\
\omega \xi & =-2 B A k, \\
0 & =-q^{2}+k^{2} .
\end{aligned}
$$

And from here, the dispersion relation is obtained

$$
\omega_{\phi}(q)=-2 B|q|\left(\gamma q^{2}+\kappa q^{4}\right),
$$

where we have introduced $\omega_{\phi}=\omega$ to specify that this result corresponds to the PF model, (7).

The derivation of the macroscopic equations of the NS$\mathrm{PF}$ model is complex due to the existing coupling between both equations. However, one can assume the hypothesis of a nonuniform mobility which effectively acts as a force propagator, mimicing the moment transfer induced by the fluid fluxes [52]. Within this picture, the mobility might be proportional to the Oseen tensor, $M\left(\mathbf{x}, \mathbf{x}^{\prime}\right)=$ $(1 / 2) \Lambda\left(\mathbf{x}, \mathbf{x}^{\prime}\right)$. In the free space the Oseen tensor is given by

$$
\Lambda\left(\mathbf{x}, \mathbf{x}^{\prime}\right)=\frac{1}{8 \pi \eta\left|\mathbf{x}-\mathbf{x}^{\prime}\right|}\left(\mathbb{1}+\frac{\left(\mathbf{x}-\mathbf{x}^{\prime}\right)\left(\mathbf{x}-\mathbf{x}^{\prime}\right)}{\left|\mathbf{x}-\mathbf{x}^{\prime}\right|^{2}}\right),
$$

where $\eta$ is the fluid viscosity. The Fourier transform of (56) is $\Lambda(q)=(\mathbb{1}-\hat{q} \hat{q}) / \eta q^{2}$, so that combined with expression (55) the relaxation rate for the NS-PF model is proportional to $\left(\gamma q+\kappa q^{3}\right)$. This is the classic result for interfaces immersed in fluids, and the formal expression reads $[52,53]$,

$$
\omega_{\eta}(q)=-\frac{1}{4 \eta}\left(\gamma q+\kappa q^{3}\right)
$$





Fig. 2. Dispersion relation for interfaces dominated by surface tension and bending (membranes), in the PF (A) and NS-PF (B) models. The simulations are perfomed in a box of constant dimensions, and hence $q_{0}$ represents the lowest mode. $w_{0}$ corresponds to the relaxation rate of the mode $q_{0}$ for each case; e.g., for the PF model of surface tension, $\omega_{0}=2 B \gamma q_{0}^{3}$, and analogously for the other three cases. Grey bold lines correspond to the theoretical prediction for the relaxation rate given by expressions (55) and (57), respectively. Both models agree with the theoretical prediction, though the curves deviate at low $q$, probably as a consequence of a finite-system effect (see main text). The fitting of the numerical results to the theoretical curves provides a measure of the effective value of the elastic parameters. We obtain $\gamma_{\phi} / \gamma_{t h}=1.02$ and $\kappa_{\eta} / \kappa_{t h}=0.88$ for the PF model, and $\gamma_{\eta} / \gamma_{t h}=1.17$ and $\kappa_{\eta} / \kappa_{t h}=0.85$ for the NS-PF model.

In order to validate the complete NS-PF model, and confirm that it captures the correct membrane dynamics given by expression (57), we numerically study the relaxation of an interface. The numerical scheme used for these tests consists in a lattice-Boltzmann implementation of the NS-PF model [54]. For further details of the model see Kendon et al. [55] and Lázaro et al. [36]. The procedure to measure the relaxation rate is analogous to the derivation outlined above. A flat interface is perturbed with an initial sinusoidal profile $h(x, 0)$. The interface position $h(x, t)$ is tracked during the evolution and, from here, the relaxation rate $\omega_{\eta}$ can be easily obtained. The initial amplitude must be small compared to the wavelength of the perturbation, $q \xi \ll 1$. In fig. 2 we show the dispersion relation for interfaces dominated by surface tension and bending (membranes), comparing the results for the PF model, given by (55), and the NS-PF model, (57). In these simulations, the size of the box is kept constant but sweeping along an extensive range of wave vectors $q$; we fix the reference mode $q_{0}$ as the one corresponding to the domain length (i.e. the minimum $q$ studied). The relaxation rates are normalized by the relevant relaxation rate $\omega_{0}$ of the mode $q_{0}$; for instance, for the PF model of surface tension, $\omega_{0}=2 B \gamma q_{0}^{3}$, and analogously for the other three cases. The dimensionless curves obtained are therefore universal for each model and type of interface.

The models reproduce the expected behaviour, though we observe a certain deviation from the theoretical prediction for the longest modes. This is likely related with the penetration length of the perturbation, $1 / k$, which scales linearly with the wavelength $\lambda=2 \pi / q$, so that the interface effectively interacts with the boundaries of the system in the limit of low $q$. By fitting the numerical results to the relaxation rate predicted by the linear theory, the ef- fective elastic moduli of each interface can be obtained. Our results show a relatively good accuracy, obtaining $\gamma_{\phi} / \gamma_{t h}=1.02$ and $\kappa_{\eta} / \kappa_{t h}=0.88$ for the PF model, and $\gamma_{\eta} / \gamma_{t h}=1.17$ and $\kappa_{\eta} / \kappa_{t h}=0.85$ for the NS-PF model. In these expressions the subscript th indicates the theoretical value of the elastic modulus as introduced in the free energy. This procedure can be extended to more complex geometries in which the influence of the membrane curvature plays an important role, a problem not well understood [56].

\section{Conclusions}

We have analyzed a phase-field model for the study of fluid membranes, and following the classic formalism of the theory of elasticity we have derived the expressions for the stress tensor, lateral stress profile and elastic moduli in terms of the order parameter. The results presented in this article demonstrate the robust physical basis of the phase-field method and its conciliation with the more familiar theory of membranes.

Once we have asserted how to relate the macroscopic material parameters of the membrane from the internal stresses of the diffuse interface, the macroscopic equations can be derived. In equilibrium, the model recovers the classic Ou Yang-Helfrich equation. Besides, we study the out-of-equilibrium behaviour of the membrane for two different dynamic models, which corresponds to a membrane immersed in a diffusive media and a membrane immersed in a liquid. We have performed a linear stability analysis that has allowed us to study the relaxational behaviour of a membrane and characterize its dynamical features. 
The methodology presented here offers the necessary tools to study membrane elastic behaviour in arbitrary geometries, showing the flexibility of the phase-field framework. In addition, the phase-field model can be extended to more general membranes (such as asymmetric or multicomponent membranes) and if a Gaussian contribution is incorporated, the method could be used to conduct studies about topological changes in membranes (e.g. fusion or vesiculization).

AHM gratefully acknowledges partial finantial support from MINECO for funding under grants FIS2013-47949-C2-1-P and FIS2016-78883-C2-1-P and DURSI for funding under grant 2014SGR878. GRL also thanks Generalitat de Catalunya for financial support under grant FI-DGR2011. IP acknowledges support from MINECO (Spain), Project FIS2015-67837-P, DURSI Project 2014SGR-922, and Generalitat de Catalunya under Program ICREA Acadèmia.

\section{Author contribution statement}

Author contribution statement: GRL, IP and AHM designed and performed the research and wrote the manuscript.

Open Access This is an open access article distributed under the terms of the Creative Commons Attribution License (http://creativecommons.org/licenses/by/4.0), which permits unrestricted use, distribution, and reproduction in any medium, provided the original work is properly cited.

\section{Appendix A. Energetic derivation of the elastic moduli}

In order to derive the expressions of the elastic moduli following an energetic argument, we consider a membrane with cylindrical and spherical geometry in which the radius is very large, so that the radial coordinate close to the interface matches the normal component of a flat membrane, $z=r-R$. Note that in this section $r$ represents the radial coordinate in the cylindrical/spherical geometry, and not the curvilinear geometry from appendix A. We consider the general expression for the membrane energy, eq. (24), that has been expanded in powers the curvature, $1 / R$, for a spherical and cylindrical distortion as shown in eq. (26). Comparing the terms of the expansion with the Helfrich free energy for the cylindrical and spherical deformations, and expanding the order parameter profile in powers of $1 / R$ as shown in eq. (25), we can express the interfacial elastic moduli in terms of the amplitudes, $\mathcal{L}_{i},(i=0,1,2)$ as depicted in eqs. (27)-(28). To lowest order, all the contributions in the expansion in $1 / R$, corresponding to the planar interface, coincide for a cylindrical and spherical deformation; for example, $\phi_{0, c}=\phi_{0, s}=\phi_{0}$. For the sake of simplicity, hereafter this subscript is only included if the geometry is relevant, whereas it is omitted for general geometries.
In order to arrive at explicit expressions for the elastic coefficients in terms of the order parameter profiles, we need to relate $\phi_{c}$ and $\phi_{s}$, which we accomplish through the Euler-Lagrange equation, eq. (3). It can be written down as

$$
\begin{aligned}
& f^{\prime}-2 g \phi^{\prime \prime}-g^{\prime}\left(\phi^{\prime}\right)^{2}-\frac{2 g}{r} \phi^{\prime}+2 c \phi^{\prime \prime \prime \prime}+\frac{4 c}{r} \phi^{\prime \prime \prime}+\mu_{\Delta}=0 \\
& f^{\prime}-2 g \phi^{\prime \prime}-g^{\prime}\left(\phi^{\prime}\right)^{2}-\frac{4 g}{r} \phi^{\prime}+2 c \phi^{\prime \prime \prime \prime}+\frac{8 c}{r} \phi^{\prime \prime \prime}+\mu_{\Delta}=0
\end{aligned}
$$

for cylindrical and spherical deformations, respectively. In the previous expressions $\psi^{\prime}(\phi)$ refers to the derivative of a function $\psi$ with respect to its variable, $\phi$. If we make use of the expansion of the order parameter around a planar interface in terms of $1 / R$, eq. (25), at first order we get

$$
\begin{aligned}
& f_{0}^{\prime} \phi_{1, c}^{\prime}-2 g_{0} \phi_{1, c}^{\prime \prime}-2 g_{0}^{\prime} \phi_{1, c}^{\prime} \phi_{0}^{\prime \prime}-2 g_{0}^{\prime} \phi_{1, c}^{\prime} \phi_{0}^{\prime} \\
& -g_{0}^{\prime} \phi_{1, c}^{\prime}\left(\phi_{0}^{\prime}\right)^{2}+2 c \phi_{1, c}^{\prime \prime \prime \prime}=2 g_{0} \phi_{0}^{\prime}-4 c \phi_{0}^{\prime \prime \prime},
\end{aligned}
$$

for the cylindrical deformation, and

$$
\begin{aligned}
& f_{0}^{\prime} \phi_{1, s}^{\prime}-2 g_{0} \phi_{1, s}^{\prime \prime}-2 g_{0}^{\prime} \phi_{1, s}^{\prime} \phi_{0}^{\prime \prime \prime}-2 g_{0}^{\prime} \phi_{1, s} \phi_{0}^{\prime} \\
& -g_{0}^{\prime} \phi_{1, s}^{\prime}\left(\phi_{0}^{\prime}\right)^{2}+2 c \phi_{1, s}^{\prime \prime \prime \prime}=2\left(2 g_{0} \phi_{0}^{\prime}-4 c \phi_{0}^{\prime \prime \prime}\right),
\end{aligned}
$$

for the spherical case. Comparing both equations one directly obtains $2 \phi_{1, c}=\phi_{1, s}$. Finally, comparing the terms to second order one gets, $4 \phi_{2, c}=\phi_{2, s}$.

In order to expand a free energy in powers of the interface deformation with respect to a planar shape, we must expand both a function, $\psi(\phi)$ of the order parameter

$$
\psi(\phi)=\psi_{0}+\frac{1}{R} \psi_{0}^{\prime} \phi_{1, c / s}+\frac{1}{R^{2}}\left[\psi_{0}^{\prime} \phi_{2, c / s}+\frac{1}{2} \psi_{0}^{\prime \prime} \phi_{1, c / s}^{2}\right]+\ldots,
$$

where we have introduced the notation $\psi\left(\phi_{0}\right)=\psi_{0}$, and where $c / s$ refers to the membrane deformation (cylinder or sphere, respectively). Moreover, the differential operators can be expanded as

$$
\begin{aligned}
\nabla \phi= & \phi_{0}^{\prime}+\frac{1}{R} \phi_{1, c / s}^{\prime}+\frac{1}{R^{2}} \phi_{2, c / s}^{\prime}+\ldots \\
\nabla^{2} \phi= & \phi_{0}^{\prime \prime}+\frac{1}{R}\left[\phi_{1, c / s}^{\prime \prime}+d \phi_{0}^{\prime}\right] \\
& +\frac{1}{R^{2}}\left[\phi_{2, c / s}^{\prime \prime}+d \phi_{1, c / s}^{\prime}-d z \phi_{0}^{\prime}\right]+\ldots \\
\nabla^{4} \phi= & \phi_{0}^{\prime \prime \prime \prime}+\frac{1}{R}\left[\phi_{1, c / s}^{\prime \prime \prime \prime}+2 d \phi_{0}^{\prime \prime \prime}\right] \\
& +\frac{1}{R^{2}}\left[\phi_{2, c / s}^{\prime \prime \prime}+2 d \phi_{1, c / s}^{\prime \prime \prime}-2 d z \phi_{0}^{\prime \prime \prime}+d(d-2) \phi_{0}^{\prime \prime}\right]+\ldots
\end{aligned}
$$

where we have used that $1 / r=1 / R[1-z / R+\ldots]$, and $d=1(2)$ for a cylindrical (spherical) deformation.

For a general free energy, as the one given in eq. (2), inserting the expanded order parameter and differential 
operators, we can compute $\mathcal{L}_{1, c / s}$ and $\mathcal{L}_{2, c / s}$ for a cylindrical/spherical deformation,

$$
\begin{aligned}
& \mathcal{L}_{1, c / s}=\frac{\partial \mathcal{L}_{c / s}}{\partial(1 / R)}=f_{0}^{\prime} \phi_{1, c / s}+g_{0}^{\prime} \phi_{1, c / s}\left(\phi_{0}^{\prime}\right)^{2}+2 g_{0} \phi_{0}^{\prime} \phi_{1, c / s}^{\prime} \\
& +2 c\left(\phi_{0}^{\prime \prime} \phi_{1, c / s}^{\prime \prime}+d \phi_{0}^{\prime \prime} \phi_{0}^{\prime}\right)+\mu_{\Delta, 1} \phi_{0}, \\
& \mathcal{L}_{2, c / s}=\frac{\partial^{2} \mathcal{L}_{c / s}}{\partial(1 / R)^{2}}=f_{0}^{\prime} \phi_{2, c / s}+\frac{1}{2} f_{0}^{\prime \prime} \phi_{1, c / s}^{2}+\frac{1}{2} g_{0}^{\prime \prime} \phi_{1, c / s}^{2}\left(\phi_{0}^{\prime}\right)^{2} \\
& +g_{0}^{\prime} \phi_{2, c / s} \phi_{0}^{\prime}+g_{0}\left(\phi_{1, c / s}^{\prime}\right)^{2}+2 g_{0} \phi_{0}^{\prime} \phi_{2, c / s}^{\prime}+2 g_{0}^{\prime} \phi_{0}^{\prime} \phi_{1, c / s}^{\prime} \phi_{1, c / s} \\
& +c\left[\left(\phi_{1, c / s}^{\prime \prime}\right)^{2}+d^{2}\left(\phi_{0}^{\prime}\right)^{2}+2 d \phi_{0}^{\prime} \phi_{1, c / s}^{\prime \prime}+2 \phi_{0}^{\prime \prime} \phi_{2, c / s}^{\prime \prime}\right. \\
& \left.+2 d \phi_{1, c / s}^{\prime} \phi_{0}^{\prime \prime}-2 z \phi_{0}^{\prime \prime} \phi_{0}^{\prime}+\mu_{\Delta, 2} \phi_{0}+\mu_{\Delta, 1} \phi_{1}\right],
\end{aligned}
$$

where $\mu_{\Delta}$ is also expanded in powers and $1 / R$ and we have made use of the fact that $\mu_{\Delta, 0}=0$. The equilibrium profiles for the distorted interface must satisfy the EulerLagrange equation, eq. (3). Expanding it in powers of $1 / R$, we get

$$
\begin{aligned}
& f_{0}^{\prime \prime} \phi_{1, c / s}-2 g_{0} \phi_{1, c / s}^{\prime \prime}-2 d g_{0} \phi_{0}^{\prime}-2 g_{0}^{\prime} \phi_{1, c / s} \phi_{0}^{\prime \prime}-g_{0}^{\prime \prime}\left(\phi_{0}^{\prime}\right)^{2} \phi_{1} \\
& -2 g_{0}^{\prime} \phi_{0}^{\prime} \phi_{1, c / s}^{\prime}+2 c\left(\phi_{1, c / s}^{\prime \prime \prime \prime}+2 d \phi_{0}^{\prime \prime \prime}\right)+\mu_{\Delta, 1}=0 .
\end{aligned}
$$

We have already shown that to lowest order, $\mathcal{L}_{0}$ is equivalent to the excess pressure and hence that the mechanical and energetic expressions for the surface tension coincide, independently of the deformation applied to the interface. Accordingly, the expressions for the surface tension obtained for both deformations coincide, as shown in eqs. (27)-(28).

The explicit expressions for the elastic moduli will depend, in general, on the choice of the dividing interface. We will consider the equimolar surface as the dividing surface, located at $z=0$,

$$
\int \mathrm{d} z\left[\phi(z)-\phi_{\text {bulk }}(z)\right]=0 .
$$

For this choice, the reference bulk densitiy reads

$$
\phi_{\text {bulk }}(z)=\phi_{-\infty} \theta(-z)+\phi_{\infty} \theta(z),
$$

where $\phi_{\infty}$ and $\phi_{-\infty}$ are the values of the concentration in the bulk regions at $z= \pm \infty$, while $\theta(z)$ stands for the Heaviside function. Expanding in the inverse of the radius of curvature $R$, the choice of the equimolar dividing surface introduces integral relations between the density fields at different powers in the expansion in the inverse of the membrane deformation. To lowest order in powers of $1 / R$ one finds

$$
\begin{aligned}
& \int\left[\phi_{0}(z)-\phi_{0, \text { bulk }}(z)\right] \mathrm{d} z=0 \\
& \int\left[\phi_{1}(z)-\phi_{1, \text { bulk }}(z)\right] \mathrm{d} z=-d \int \mathrm{d} z\left[z\left(\phi_{0}(z)-\phi_{0, \text { bulk }}(z)\right)\right] .
\end{aligned}
$$

The expressions for the spontaneous curvature, and bending and Gaussian moduli require some algebra. Introducing (3) and (A.9) in (A.7) and (A.8), the elastic moduli can be rewritten. The spontaneous curvature reduces to

$$
-\kappa C_{0}=\int z \mathcal{L}_{0} \mathrm{~d} z,
$$

which amounts to stating that $\int \mathcal{L}_{1} \mathrm{~d} z=0$, showing that the expression for $\kappa C_{0}$ coincides for a spherical and cylindrical deformation and with the expression obtained mechanically. For the bending rigidity we must focus on the cylindric geometry, as bending and Gaussian rigidities are coupled in the spherical case. Integrating the expressions for the energy coefficients (A.7) and (A.8), it can be shown that the bending rigidity takes the form

$$
\kappa=\int\left(z \mathcal{L}_{1, c}+3 c \phi_{0}^{\prime 2}\right) \mathrm{d} z
$$

where the term associated with $\phi_{1, c / s}$ represents a perturbative contribution due to the bending of the interface.

The bending rigidity (A.13) must be introduced in the expression for the Gaussian rigidity in (28), and also considering the relations between $\phi_{s}$ and $\phi_{c}$ one obtains

$$
\kappa_{G}=\int\left[z^{2} \mathcal{L}_{0}-2 c\left(\phi_{0}^{\prime}\right)^{2}\right] \mathrm{d} z .
$$

It can be shown that, to leading order, eqs. (A.12), (A.13), (A.14) agree with the results found by Gompper and Zschocke [12]. The link between the reference free energy density $\mathcal{L}_{0}$ and the lateral stress profile (19) requires computing of the first integral of the Euler-Lagrange equation. Making use of eq. (30), the relation between $\mathcal{L}_{0}$ and $s_{\phi}$ is easily deduced

$$
\begin{aligned}
\int \mathcal{L}_{0} \mathrm{~d} z & =\int\left[f_{0}+g_{0}\left(\phi_{0}^{\prime}\right)^{2}+c\left(\phi_{0}^{\prime \prime}\right)^{2}\right] \mathrm{d} z \\
& =\int\left[2 g_{0}\left(\phi_{0}^{\prime}\right)^{2}+2 c\left(\partial_{0}^{\prime \prime}\right)^{2}-2 c \phi_{0}^{\prime} \phi_{0}^{\prime \prime}\right] \mathrm{d} z \\
& =\int s_{\phi}(z) \mathrm{d} z .
\end{aligned}
$$

Multiplying by $\phi_{0}^{\prime}$ eq. (A.9), and integrating over space, one can show that all terms proportional to $\phi_{1}$ vanishes, leading to and expression for

$$
\mu_{\Delta, 1 c} \int_{-\infty}^{\infty} \frac{\mathrm{d} \phi_{0}}{\mathrm{~d} z} \mathrm{~d} z=\gamma
$$

and comparing the expression for the spontaneous curvature for the spherical and cylindrical deformations, $\mu_{\Delta, 1 s}=2 \mu_{\Delta, 1 c}$. The derivations shows that the change in the reference thermodynamic state as the interface is curved, quantified by $\mu_{\Delta}$, does not affect the expressions for the elastic coefficients.

\section{Appendix B. The sharp interface limit}

The sharp interface limit is a classic method for determining the macroscopic equations of diffuse interface models [48]. It exploits the separation between the length scale of the interface, given by $\epsilon$, and the typical length of the interface, which can be defined from the total curvature as $l \sim 1 / C$. The limit applies only in the low-curvature limit, $\epsilon C \ll 1$. 


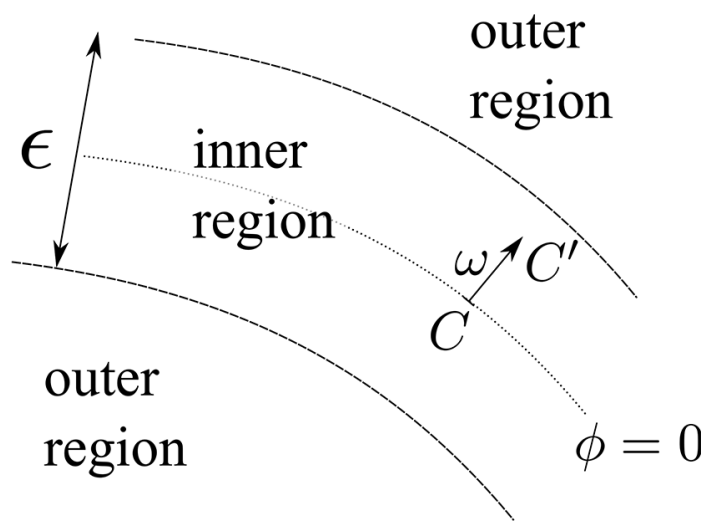

Fig. 3. Scheme of the sharp interface method. The interface has a normal direction at each point given by the coordinates $r$ in the outer region and the fast coordinate $\omega=r / \epsilon$ in the inner region. In the inner region, each isosurface $\phi=$ const. has an associated mean curvature $C^{\prime}(u, s)$, whereas the isosurface $\phi=0$ is associated to $C$.

\section{Appendix B.1. The inner and the outer region}

The interface is described by the curvilinear coordinates $(r, s, u)[19]$. These coordinates represent a naïve parametrization of the membrane surface. The vector basis is chosen as follows. At each position of the surface, the normal vector $\mathbf{n}$ is defined. From the tangential plane we choose two vectors forming an orthonormal trihedron, $\mathbf{t}_{2}=\mathbf{n} \times \mathbf{t}_{1}$. We define a vector position $\mathbf{n}$ that can be decomposed into the vector position in the surface and the normal projection, $\mathbf{r}=\mathbf{R}(s, u)+r \mathbf{n}(s, u)$, where $r$ is the coordinate in the direction of $\mathbf{n}$ and $(s, u)$ are the coordinates in the directions of $\mathbf{t}_{1}$ and $\mathbf{t}_{2}$, respectively. Hence, $(r, s)$ are orthogonal coordinates on the membrane surface, and describe arclengths of the curve formed by the intersection of the membrane surface and the planes $(r, s)=$ const. and $(r, u)=$ const. $r$ is normal to the interface at each point $(s, u)$ on the surface. A scheme of the interface decomposition is provided in fig. 3 .

The space is separated into two different regions. The interfacial region, called here inner region, is described by a fast coordinate $\omega=r / \epsilon$. Thus, the interfacial region is zoomed up in order to resolve the details of the smooth interface, although in the outer region the interface is effectively sharp in the limit $\epsilon \rightarrow 0$. The starting point is a flat interface in equilibrium, with relaxed profile $\phi_{0}$. If a small perturbation is induced, the deviations from the equilibrium profile can be expanded in terms of $\epsilon$,

$$
\begin{aligned}
& a(\omega, s, u)=a_{0}(\omega, s, u)+\epsilon a_{1}(\omega, s, u)+\epsilon^{2} a_{2}(\omega, s, u)+\ldots \\
& A(r, s, u)=A_{0}(r, s, u)+\epsilon A_{1}(r, s, u)+\epsilon^{2} A_{2}(r, s, u)+\ldots
\end{aligned}
$$

where capital letters denote variables in the outer region, and lower case letters indicate variables in the inner region. In the limit $\omega \rightarrow \pm \infty$ both regions meet, and the matching conditions read

$$
\begin{aligned}
a_{0}(\omega, s, u)= & A_{0}\left(0^{ \pm}, s, u\right) \\
a_{1}(\omega, s, u)= & a_{1}\left(0^{ \pm}, s, u\right)+\omega \partial_{r} a_{0}\left(0^{ \pm}, s, u\right), \\
a_{2}(\omega, s, u)= & a_{2}\left(0^{ \pm}, s, u\right)+\omega \partial_{r} a_{1}\left(0^{ \pm}, s, u\right) \\
& +\frac{\omega^{2}}{2} \partial_{r r}^{2} a_{1}\left(0^{ \pm}, s, u\right), \cdots
\end{aligned}
$$

The dynamic equation (7) is analogously decomposed in the two regions, with the corresponding inner and outer variables. The differential operators must be also expanded in terms of $\epsilon$. We also assume that the interface motion is much larger than the characteristic timescale of order parameter diffusion, and hence the time is also rescaled $\tau=\epsilon t$, where $\tau$ is the time in the inner region. Hence, in this quasiestatic approximation we can write

$$
\partial_{t} \phi(r)=\epsilon \partial_{\tau} \phi(\omega)-\frac{1}{\epsilon} \partial_{\tau} \omega \partial_{\omega} \phi(\omega)
$$

and, defining the normal velocity of the interface $v=$ $-\partial_{\tau} \omega$, the dynamic equation reads

$$
\epsilon \partial_{\tau} \phi-\frac{1}{\epsilon} v \partial_{\omega} \phi=M \nabla^{2} \mu
$$

where the Laplacian must be expanded, as shown below. The method does not allow for finding explicit solutions of the fields, but provide a set of equations that in the limit $\epsilon \rightarrow 0$ represent the macroscopic equations of the model.

\section{Appendix B.2. Differential operators}

As previously stated, the differential operators must be also expanded in terms of $\epsilon$. In the inner coordinates, $\nabla \phi=\left(\epsilon^{-1} \partial_{\omega} \phi, \partial_{s} \phi, \partial_{u} \phi\right)$, and thus at leading order only the normal coordinate contributes. This means that the variations of $\phi$ along the coordinate directions $(s, u)$ are disregardable with respect to variations in $\omega$. For simplicity, in the subsequent calculations we deliverately neglect some derivatives of the tangential coordinates, which do not contribute after the expansion, in order to simplify the expressions obtained. Additionally, for the sake of clarity we perform all the calculations in terms of the coordinate $r$ and only introduce $\omega$ at the end of the calculations. Some useful identities are [22]

$$
\begin{aligned}
& \hat{n}=\nabla r, \\
& C=-\nabla \cdot \hat{n}=-\nabla^{2} r, \\
& G=-(1 / 2)\left[2 \operatorname{tr}\left(\left(\nabla_{\alpha} \nabla_{\beta} r\right)^{2}\right)-\left(\operatorname{tr}\left(\nabla_{\alpha} \nabla_{\beta} r\right)\right)^{2}\right],
\end{aligned}
$$

for the normal vector and total curvature, respectively. Note that, because the normal vector is unitary, $(\nabla r)^{2}=$ 1 , and consequently

$$
n_{\alpha} \nabla_{\beta} n_{\alpha}=(1 / 2) \nabla_{\beta}\left(n_{\alpha} n_{\alpha}\right)=0 .
$$

We suppose that at each point of the isosurface $\phi=$ const., there is a local coordinate system given by the coordinate $r(\mathbf{x})$, normal to the surface, and the tangential 
coordinates $(s(\mathbf{x}), u(\mathbf{x}))$. We suppose that there exists a solution for the order parameter profile of the form $\phi=$ $\phi(r, s, u)$. We can write the gradient and Laplacian as

$$
\begin{aligned}
\nabla \phi= & \partial_{r} \phi \nabla r+\partial_{s} \phi \nabla s+\partial_{u} \phi \nabla u . \\
\nabla^{2} \phi= & \partial_{r}^{2} \phi(\nabla r)+\partial_{r} \phi \nabla^{2} r+\partial_{s}^{2} \phi(\nabla s)^{2}+\partial_{s} \phi \nabla^{2} s \\
& +\partial_{u}^{2} \phi(\nabla u)^{2}+\partial_{u} \phi \nabla^{2} u .
\end{aligned}
$$

For simplicity, we only consider the terms associated to the higher derivative, e.g., $\partial_{s}^{2}$ in the Laplacian. Introducing these considerations, the expression for the Laplacian can be rewritten as

$$
\nabla^{2} \phi=\partial_{r}^{2} \phi-C^{\prime} \partial_{r} \phi+\partial_{s}^{2} \phi+\partial_{u}^{2} \phi+\cdots .
$$

Here, $C^{\prime}$ corresponds to the local total curvature of the isosurface at each point of the space $(r, u, s)$. However, in the interface region it is convenient to write the local curvature of the isosurface $\phi=$ const. expressed in terms of the curvature of the isosurface $\phi=0$, which we denote $C$, given that in the sharp interface limit $C^{\prime} \rightarrow C$. The relation between the curvature of the two isosurfaces is given by

$$
C^{\prime}=C\left[\frac{2 r G / C-1}{1-r C+r^{2} G}\right] .
$$

Introducing this expression in (B.8), the expression for the Laplacian is given by

$$
\nabla^{2} \phi=\partial_{r}^{2} \phi-\left[\frac{2 r G-C}{1-r C+r^{2} G}\right] \partial_{r} \phi+\partial_{s}^{2} \phi+\partial_{u}^{2} \phi+\cdots .
$$

At low curvatures, the term in brackets associated with $\partial_{r} \phi$ can be expanded, obtaining

$$
\begin{aligned}
\nabla^{2} \phi= & \partial_{r}^{2} \phi-C+\left[r\left(C^{2}-2 G\right)+r^{2}\left(C^{3}-3 G C+\cdots\right)\right] \partial_{r} \phi \\
& +\partial_{s}^{2}+\partial_{u}^{2}+\cdots .
\end{aligned}
$$

If the fast coordinate $\omega$ is introduced, the expression reads

$$
\begin{aligned}
\nabla^{2} \phi= & \epsilon^{-2} \partial_{\omega}^{2} \phi-\epsilon^{-1}\left[C+\epsilon \omega\left(C^{2}-2 G\right)\right. \\
& \left.+\epsilon^{2} \omega^{2}\left(C^{3}-3 G C+\ldots\right)\right] \partial_{\omega} \phi+\partial_{s}^{2} \phi+\partial_{u}^{2} \phi+\cdots
\end{aligned}
$$

The derivation of the fourth derivative, $\nabla^{4}=\nabla^{2} \nabla^{2}$ is more complicated. By taking the derivative of the expression of the Laplacian (B.7), one obtains

$$
\begin{aligned}
\nabla^{2} \nabla^{2} \phi= & \partial_{r}^{4} \phi+2 \nabla^{2} r \partial_{r}^{3} \phi+2 \nabla_{\alpha} r \nabla_{\alpha} \nabla^{2} r \partial_{r}^{2} \phi \\
& +\left(\nabla^{2} r\right)^{2} \partial_{r}^{2} \phi+\nabla^{2} \nabla^{2} r \partial_{r} \phi .
\end{aligned}
$$

In this expression, several terms can be readily identified in terms of the total curvature $\left(\right.$ e.g. $\left.C^{\prime 2}=\left(\nabla^{2} r\right)^{2}\right)$, but particularly the physical meaning of $\nabla_{\alpha} r \nabla_{\alpha} \nabla^{2} r$ is not straightforward.

For convenience, we perform below some algebraic manipulations that will be useful in the derivation of the equilibrium condition in sect. 4 . Let us consider first the fourth term in the right-hand side of (B.13). Multiplying by $\partial_{r} \phi$ and $\left(\nabla_{\alpha} r\right)\left(\nabla_{\alpha} r\right)=1$, and integrating by parts

$$
\begin{aligned}
& \int \nabla_{\alpha} r \nabla_{\alpha} r \partial_{r} \phi \partial_{r}^{2} \phi\left(\nabla^{2} r\right)^{2} \mathrm{~d} x= \\
& -\frac{1}{2} \int\left[\left(\nabla^{2} r\right)^{3}+2 \nabla_{\alpha} r \nabla_{\alpha} \nabla^{2} r \nabla^{2} r\right]\left(\partial_{r} \phi\right)^{2} \mathrm{~d} x .
\end{aligned}
$$

The interpretation of this expression requires to consider the identity $C^{\prime 2}-4 G^{\prime}=2 \operatorname{tr}\left(\nabla^{2} r\right)^{2}-\left(\nabla^{2} r\right)^{2}$, as can be obtained from identities (B.5). Then, it is straightforward to show the equivalence:

$$
\begin{aligned}
C^{\prime}\left(C^{\prime 2}-4 G^{\prime}\right)= & -\nabla^{2} r\left(2 \operatorname{tr}\left(\nabla_{\alpha} \nabla_{\beta} r\right)^{2}-\left(\nabla^{2} r\right)^{2}\right) \\
= & -2 \nabla_{\alpha}\left(\nabla^{2} r \nabla_{\beta} r \nabla_{\alpha} \nabla_{\beta} r\right) \\
& +2\left(\nabla_{\alpha} \nabla^{2} r \nabla_{\beta} r \nabla_{\alpha} \nabla_{\beta} r\right) \\
& +2 \nabla^{2} r \nabla_{\alpha} r \nabla_{\alpha} r \nabla^{2} r+\left(\nabla^{2} r\right)^{3} \\
= & \left(\nabla^{2} r\right)^{3}+2 \nabla_{\alpha} r \nabla^{2} r \nabla_{\alpha} \nabla^{2} r,
\end{aligned}
$$

where, in the last equality, we have used (B.6). The comparison between eqs. (B.14) and (B.15) demonstrates that the fourth term in (B.13) relates with $-(1 / 2) C^{\prime}\left(C^{\prime 2}-\right.$ $\left.4 G^{\prime}\right)$.

Considering now the third term in the right-hand side of (B.13), multiplying by $\partial_{r} \phi$ and $\left(\nabla_{\beta} r\right)\left(\nabla_{\beta} r\right)=1$,

$$
\begin{aligned}
& \int \nabla_{\beta} r \nabla_{\beta} r \nabla_{\alpha} r \nabla_{\alpha} \nabla^{2} r \partial_{r}^{2} \phi \partial_{r} \phi \mathrm{d} x=-\frac{1}{2} \int\left[\nabla_{\beta} r \nabla_{\beta} \nabla^{2} r \nabla^{2} r\right. \\
& \left.+\nabla_{\beta} r \nabla_{\alpha} \nabla_{\beta} r \nabla_{\alpha} \nabla^{2} r+\nabla_{\beta} r \nabla_{\alpha} r \nabla_{\alpha} \nabla_{\beta} \nabla^{2} r\right]\left(\partial_{r} \phi\right)^{2} \mathrm{~d} x .
\end{aligned}
$$

Note that the second term in the expression in brackets vanishes by (B.6). From the expression for the gradient operator projected over the surface $S, \nabla_{\alpha}^{S}=\nabla_{\alpha}-n_{\alpha} n_{\beta} \nabla_{\beta}$, the Laplace-Beltrami operator over the surface reads

$$
\Delta_{S}=\nabla_{\alpha}^{S} \nabla_{\alpha}^{S}=\nabla^{2}+C^{\prime} n_{\alpha} \nabla_{\alpha}-n_{\alpha} n_{\beta} \nabla_{\alpha} \nabla_{\beta} .
$$

Manipulating this expression, and using $n_{\alpha}=\nabla_{\alpha} r$, leads to,

$$
\begin{aligned}
\Delta_{S} C^{\prime} & =\nabla^{2} C^{\prime}+C^{\prime} n_{\alpha} \nabla_{\alpha} C^{\prime}-n_{\alpha} n_{\beta} \nabla^{\alpha} \nabla^{\beta} C^{\prime} \\
& =-\nabla^{2} \nabla^{2} r+\nabla^{2} r \nabla_{\alpha} r \nabla_{\alpha} \nabla^{2} r+\nabla_{\alpha} r \nabla_{\beta} r \nabla_{\alpha} \nabla_{\beta} \nabla^{2} r .
\end{aligned}
$$

Hence, our calculations show that the third and fifth terms of (B.13) are equivalent to the surface variations of the curvature, $\Delta_{S} C^{\prime}$. As a summing up, introducing the fast coordinate $\omega$ and multiplying equation (B.13) by $\partial_{\omega} \phi$ we obtain the relation

$$
\begin{aligned}
& \int \partial_{\omega} \phi \nabla^{2} \nabla^{2} \phi \mathrm{d} x=\int\left\{\epsilon^{-4} \partial_{\omega}^{4} \phi \partial_{\omega} \phi\right. \\
& +2\left[C+\omega\left(2 G-C^{2}\right)+\omega^{2}\left(C^{3}-3 G C\right)+\ldots\right] \partial_{\omega}^{3} \phi \partial_{\omega} \phi \\
& \left.-\frac{1}{2} \epsilon^{-1} C\left(C^{2}-4 G\right)\left(\partial_{\omega} \phi\right)^{2}-\epsilon^{-1} \Delta_{S} C\left(\partial_{\omega} \phi\right)^{2}\right\} \mathrm{d} x .
\end{aligned}
$$

Note that the terms associated with the first derivative, $\partial_{\omega} \phi$, correspond to the highest order considered in the expansion and at this order $C=C^{\prime}$. 


\section{References}

1. B. Alberts, D. Bray, M. Raff, K. Roberts, J.D. Watson, Molecular Biology of the Cell (Garland Science, New York, 1994).

2. S.A. Safran, Statistical Thermodynamics of Surfaces, Interfaces, and Membranes (Addison-Wesley, Reading, 1994).

3. E. Sackmann, Biological Membranes Architecture and Function, in Handbook of Biological Physics, edited by R. Lipowsky, E. Sackmann, Vol. 1 (Elsevier, 1995).

4. Z. Peng, X. Li, I.V. Pivkin, M. Dao, G.E. Karniakidis, S. Suresh, Proc. Natl. Acad. Sci. U.S.A. 110, 13356 (2013).

5. J. Li, M. Dao, C.T. Lim, S. Suresh, Biophys. J. 88, 3707 (2005).

6. Y. Park, C.A. Best, K. Badizadegan, R.R. Dasari, M.S. Feld, T. Kriabova, M.L. Henle, A.J. Levine, G. Popescu, Proc. Natl. Acad. Sci. U.S.A. 107, 6731 (2010).

7. V. Fleury, J.-F. Gouyet, M. Leonetti, Branching in nature (Springer-Verlag, Berlin, 2001).

8. W.J. Boettinger, J.A. Warren, C. Beckermann, A. Karma, Annu. Rev. Mater. Res. 32, 163 (2002).

9. A. Karma, D.A. Kessler, H. Levine, Phys. Rev. Lett. 87, 045501 (2001).

10. M. Alava, M. Dubé, M. Rost, Adv. Phys. 53, 83 (2004).

11. I. Steinbach, Mod. Simul. Mater. Sci. Eng. 17, 073001 (2009).

12. G. Gompper, S. Zschocke, Europhys. Lett. 16, 731 (1991)

13. G. Gompper, S. Zschocke, Phys. Rev. A 46, 4836 (1992).

14. E.M. Blokhuis, D. Bedeaux, Mol. Phys. 80, 705 (1993).

15. E.M. Blokhuis, Phys. Chem. Chem. Phys. 100, 313 (1996).

16. G.R. Lázaro, I. Pagonabarraga, A. Hernández-Machado, Chem. Phys. Lipids 185, 46 (2015).

17. D. Jamet, C. Misbah, Phys. Rev. E 78, 041903 (2008).

18. Q. Du, C. Liu, X. Wang., J. Comput. Phys. 198, 450 (2004).

19. T. Biben, K. Kassner, C. Misbah, Phys. Rev. E 72, 041921 (2005).

20. F. Campelo, A. Hernández-Machado, Eur. Phys. J. E 20, 37 (2006).

21. F. Campelo, A. Hernández-Machado, Eur. Phys. J. ST 143, 101 (2007).

22. Q. Du, C. Liu, R. RYham, X. Wang, Nonlinearity 18, 1249 (2005).

23. Q. Du, M. Li, C. Liu, Discr. Continuous Dyn. Syst. B 8, 539 (2007).

24. W. Helfrich, Amphiphilic mesophases made of defects, in Liquids at Interfaces, Les Houches Session XXXV, edited by R. Balian, M. Kleman, J. Poirier (North Holland, Amsterdam, 1981).
25. D. Marsh, Chem. Phys. Lipids. 144, 146 (2006).

26. G. Gompper, M. Schick, Self assembling amphiphilic systems (Academic Press Limited, London, 1994).

27. W. Helfrich, Z. Naturforsch. C 28, 693703 (1973).

28. M.P. Sheetz, S.J. Singer, Proc. Natl. Acad. Sci. U.S.A. 71, 4457 (1974).

29. E.A. Evans, Biophys. J. 14, 923 (1974).

30. A. Iglic, J. Biomech. 35-40, 923 (1997).

31. G.H.W. Lim, M. Wortis, R. Mukhopadhyay, Proc. Natl. Acad. Sci. U.S.A. 99, 16766 (2002).

32. F. Campelo, A. Hernández-Machado, Phys. Rev. Lett. 100, 158103 (2008).

33. G. Gompper, M. Schick, Phys. Rev. Lett. 65, 1116 (1990).

34. F. Campelo, A. Hernández-Machado, Phys. Rev. Lett. 99, 088101 (2007).

35. X. Wang, Q. Du, J. Math. Biol. 56, 347 (2008).

36. G.R. Lázaro, A. Hernández-Machado, I. Pagonabarraga, Soft Matter 10, 7195 (2014).

37. J.E. Cahn, J.E. Hilliard, J. Chem. Phys. 31, 688 (1959).

38. L.D. Landau, E.M. Lifshitz, Theory of Elasticity (Butterworth-Heinmann, Oxford, 1999).

39. J. Brannick, C. Liu, T. Qian, H. Sun, Numer. Math. Theor. Methods Appl. 8, 220 (2015).

40. J.S. Rowlinson, B. Widom, Molecular Theory of Capillarity (Butterworth, Boston, 1989).

41. W. Helfrich, M.M. Kozlov, J. Phys. II France 3, 287 (1993).

42. S. Baoukina, S.J. Marrink, D.P. Tieleman, Faraday Discuss. 144, 393 (2010).

43. D. Marsh, Biophys. J. 93, 3884 (2007).

44. M. Hu, D.H. de Jong, S.J. Marrink, M. Deserno, Faraday Discuss. 161, 365 (2013).

45. A.G. Petrov, J. Bivas, Progr. Surf. Sci. 18, 389 (1984).

46. G. Gompper, S. Klein, J. Phys. II 2, 1725 (1992).

47. E.M. Blokhuis, H.N.W. Lekkerkerker, I. Szleifer, J. Chem. Phys. 112, 6023 (2000).

48. R. Folch, J. Casademunt, A. Hernández-Machado, L. Ramírez-Piscina, Phys. Rev. E 60, 1724 (1999).

49. A. Hernández-Machado, A.M. Lacasta, E. Mayoral, E.C. Poiré, Phys. Rev. E 68, 046310 (2003).

50. Z. Ou-Yang, W. Helfrich, Phys. Rev. Lett. 59, 2486 (1987).

51. A. Hernández-Machado, D. Jasnow, Phys. Rev. A 37, 656 (1988).

52. R. Granek, J. Phys. II France 7, 1761 (1997).

53. O. Theissen, G. Gompper, D.M. Kroll, Europhys. Lett. 42, 419 (1998).

54. J.-C. Desplat, I. Pagonabarraga, P. Bladon, Comput. Phys. Commun. 134, 273 (2001)

55. V.M. Kendon, M.E. Cates, I. Pagonabrraga, J.-C. Desplat, P. Blandon, J. Fluid Mech. 440, 147 (2001).

56. H. Shiba, H. Noguchi, Phys. Rev. E 84, 031926 (2011). 